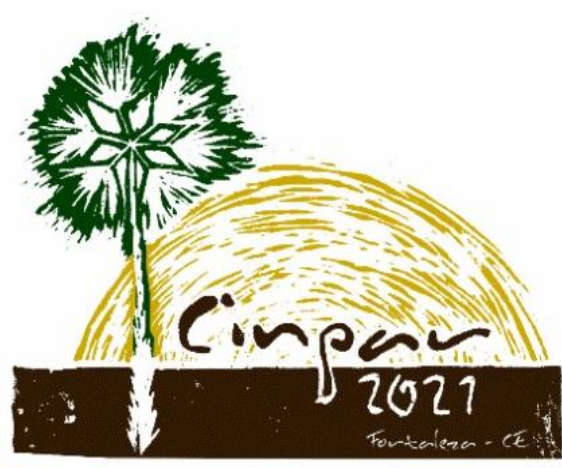

XVII Congresso Internacional sobre Patologia e Reabilitação das Construções

XVII Congreso Internacional sobre Patología y Rehabilitación de las Construcciones

XVII International Conference on Pathology and Constructions Rehabilitation

FORTALEZA (Brasil), 3 a 5 de junho de 2021

https://doi.org/10.4322/CINPAR.2021.121

\title{
Efeito do Teor de Nanossílica no Desempenho Mecânico do Microconcreto
}

\section{Effect of Nanosilica Content on the Mechanical Performance of Microconcrete}

\author{
Rafaella Lins Ribeiro ARAÚJO ${ }^{1}$, Kamyla Hellen Lima VIANA ${ }^{2}$, Yuri Sotero Bomfim FRAGA ${ }^{3}$ \\ ${ }^{1}$ Centro Universitário Euro-Americano (UNIEURO), Brasília/DF, Brasil, rafaella.lins@hotmail.com \\ ${ }^{2}$ Centro Universitário Euro-Americano (UNIEURO), Brasília/DF, Brasil, kamylaviana99@gmail.com \\ ${ }^{3}$ Centro Universitário Euro-Americano (UNIEURO), Brasília/DF, Brasil, yurisotero.engcivil@gmail.com
}

\begin{abstract}
Resumo: $\mathrm{O}$ estudo da nanotecnologia aplicada à construção civil é crescente nos últimos anos. Parte desses estudos investiga a utilização de nanossílica (NS) em compósitos cimentícios para aumento do desempenho mecânico e da durabilidade. De forma a contribuir com esses estudos, esta pesquisa teve como objetivo avaliar a influência do teor de NS em substituição ao cimento Portland no desempenho mecânico de microconcretos. Foram moldados seis traços de microconcreto, sendo uma referência com $100 \%$ cimento Portland e os demais substituindo parcialmente o cimento Portland por NS nos teores de $2 \%, 4 \%, 6 \%, 8 \%$ e $10 \%$ em relação à massa de cimento. Foi avaliada a resistência à compressão dos microconcretos aos 1, 3, 7 e 14 dias de hidratação. Observou-se que os microconcretos com $6 \%, 8 \%$ e $10 \%$ de NS resultaram em maiores resistências à compressão até os 3 dias de hidratação, porém após essa idade o microconcreto com $4 \%$ de NS resultou no maior desempenho mecânico dentre os microconcretos avaliados. Dessa forma, é recomendável a utilização de $4 \%$ de NS para o aumento no desempenho mecânico de compósitos cimentícios.
\end{abstract}

Palavras-chave: Compósitos cimentícios; Resistência à compressão; Efeito pozolânico; Nanossílica.

\begin{abstract}
The study of nanotechnology applied to civil construction has been growing in recent years. Part of these studies investigates the use of nanosilica (NS) in cementitious composites to increase mechanical performance and durability. In order to contribute to these studies, this research aimed to assess the influence of NS content in substitution of Portland cement on the mechanical performance of microconcrete. Six microconcretes were molded, one reference with $100 \%$ of Portland cement and the others partially replacing the Portland cement by NS in the contents of $2 \%, 4 \%, 6 \%, 8 \%$ and $10 \%$ in relation to the cement weight. The compressive strength of microconcrete was evaluated at 1, 3, 7 and 14 days of hydration. It was observed that the microconcrete with $6 \%, 8 \%$ and $10 \%$ of NS resulted in higher compressive strengths up to 3 days of hydration, however after that age the microconcrete with $4 \%$ of NS resulted in higher mechanical performance among microconcretes evaluated. Thus, it is recommended to use $4 \%$ NS to increase the mechanical performance of cementitious composites.
\end{abstract}

Keywords: Cementitious composites; Compressive strength; Pozzolanic effect; Nanosilica. 


\section{Introdução}

O cimento é um dos materiais mais utilizados na construção civil. Tendo em vista o crescimento desenfreado dos centros urbanos, busca-se cada vez mais por produtos mais duráveis e seguros em questões de desempenho em uso. Desta forma, a adição da nanossílica (NS) vem ganhando espaço na utilização em conjunto com o cimento e a busca e compreensão do seu comportamento tornou-se de grande relevância para análise de compósitos cimentícios (FRAGA et al., 2020).

Por ser um material com elevado grau de amorfismo, com partículas na escala nanométrica e elevada superfície específica, a adição de NS acelera o processo de hidratação do cimento através da criação de pontos de nucleação. A NS é uma adição que proporciona alta resistência e durabilidade através da alteração da microestrutura da pasta do concreto (Ll et al., 2017).

No estudo realizado por Singh et al. (2012), a adição de 5\% de NS proporcionou aumento de $64 \%$ no desempenho mecânico da pasta de cimento em relação ao traço referência sem adição com um dia de hidratação. Aos 28 dias de hidratação esse aumento foi de $35 \%$ em relação ao traço referência. De acordo com Fraga et al. (2020), esse aumento no desempenho mecânico é atribuído principalmente devido à reação pozolânica da NS com o hidróxido de cálcio $(\mathrm{CH})$ proveniente das reações de hidratação do cimento Portland, formando C-S-H adicional.

Na pesquisa realizada por Senff et al. (2010), a mesma tendência foi observada, ou seja, a adição de NS resultou no aumento da resistência inicial da pasta de cimento em relação ao traço referência. Observa-se que esse comportamento está associado com a formação de C-S-H adicional e refinamento da estrutura porosa, que contribuem para o aumento da durabilidade dos compósitos cimentícios (FRAGA et al., 2020).

Como a adição de nanossílica ocasiona o refinamento da estrutura porosa do concreto, há uma melhora considerável nos resultados mecânicos. Com isso, pode-se alcançar maior esbeltez da estrutura. A baixa porosidade diminui os efeitos da lixiviação, obtendo maior durabilidade e segurança dos materiais cimentícios (FLORES et al., 2017).

Apesar do comportamento benéfico no aumento do desempenho mecânico e da durabilidade dos compósitos cimentícios com a adição de NS, também existem comportamos não desejados oriundos da sua adição. Devido à elevada superfície específica da NS, há aumento da demanda de água ou de aditivos redutores de água para obter a mesma consistência de um concreto convencional (ABD.EL.ALEEM et. al., 2014; VASKE et al., 2010).

Outro comportamento não desejado oriundo da utilização da NS em conjunto com o cimento Portland na fabricação de pastas, argamassas e concretos é o aumento da retração. Como a adição de NS acelera as reações de hidratação e a retração dos compósitos cimentícios, ocorre o aumento das tensões de tração, podendo resultar no surgimento de fissuras e contribuindo para a diminuição do desempenho mecânico (SILVA JÚNIOR, 2017).

Diante do exposto, esta pesquisa tem como objetivo analisar o efeito do teor de NS na resistência à compressão do microconcreto nas idades iniciais, avaliando o desempenho mecânico ao longo do tempo, bem como avaliar se diferentes teores de NS possuem efeito significativo na resistência à compressão de concretos.

\section{Metodologia}

\subsection{Materiais}

Para o presente estudo, foi utilizado o cimento Portland CP V- ARI, nanossílica coloidal (NS) com $30 \%$ de teor de sólidos, agregado miúdo natural, aditivo plastificante (SP) à base de policarboxilato e água da Companhia de Saneamento Ambiental do Distrito Federal (CAESB). No Quadro 1 são mostradas as propriedades desses materiais. 
Quadro 1 - Caracterização do cimento Portland, da nanossílica e do aditivo superplastificante

\begin{tabular}{|c|c|c|}
\hline Material & Propriedade & Valores \\
\hline \multirow{4}{*}{ Cimento Portland } & \multirow{2}{*}{ Tempo de início e fim de pega (min) } & 115 \\
\hline & & 204 \\
\hline & Índice de finura na peneira $75 \mu \mathrm{m}(\%)$ & 2 \\
\hline & Massa especifica $\left(\mathrm{g} / \mathrm{cm}^{3}\right)$ & 3,15 \\
\hline \multirow{4}{*}{ Nanossílica } & Massa especifica $\left(\mathrm{g} / \mathrm{cm}^{3}\right)$ & 1,2 \\
\hline & $\mathrm{pH}$ & 10,5 \\
\hline & Teor de sólidos (\%) & 30 \\
\hline & Diâmetro médio (nm) & 22,75 \\
\hline \multirow{3}{*}{$\begin{array}{l}\text { Aditivo } \\
\text { superplastificante }\end{array}$} & Cor & Líquido alaranjado \\
\hline & Massa específica $\left(\mathrm{g} / \mathrm{cm}^{3}\right)$ & $\begin{array}{c}\text { 1,095 em relação à massa } \\
\text { específica da água }\end{array}$ \\
\hline & $\mathrm{pH}$ & 3,9 \\
\hline
\end{tabular}

O agregado miúdo utilizado foi peneirado em quatro frações granulométricas estabelecidas na NBR 7215 (ABNT, 2019), conforme mostrado na Quadro 2.

Quadro 2- Composição da areia utilizada na pesquisa (ABNT, 2019).

\begin{tabular}{|c|c|c|}
\hline Denominação da fração & $\begin{array}{c}\text { Abertura nominal de malha } \\
\text { das peneiras (mm) }\end{array}$ & $\begin{array}{c}\text { Porcentagem retida em } \\
\text { massa de material nas } \\
\text { peneiras (\%) }\end{array}$ \\
\hline Grossa & 2,4 e 1,2 & $25 \pm 5$ \\
\hline Média grossa & 1,2 e 0,6 & $50 \pm 5$ \\
\hline Média fina & 0,6 e 0,3 & $75 \pm 5$ \\
\hline Fina & 0,3 e 0,15 & $97 \pm 3$ \\
\hline
\end{tabular}

\subsection{Preparação dos microconcretos}

Para verificar o efeito de diferentes teores de NS no desempenho mecânico do microconcreto, foram estududados seis traços, sendo um referência sem adições (REF) e outros cinco contendo $2 \%, 4 \%, 6 \%, 8 \%$ e $10 \%$ de NS, conforme mostrado no Quadro 3.

Quadro 3 - Nomenclaturas com suas respectivas descrições

\begin{tabular}{|c|c|}
\hline Nomenclaturas & Descrição \\
\hline REF & Microconcreto sem a incorporação da nanossílica \\
\hline $2 \mathrm{NS}$ & Microconcreto com incorporação de 2\% nanossílica \\
\hline $4 \mathrm{NS}$ & Microconcreto com incorporação de 4\% nanossílica \\
\hline $6 \mathrm{NS}$ & Microconcreto com incorporação de 6\% nanossílica \\
\hline $8 \mathrm{NS}$ & Microconcreto com incorporação de 8\% nanossílica \\
\hline $10 \mathrm{NS}$ & Microconcreto com incorporação de 10\% nanossílica \\
\hline
\end{tabular}

A quantidade de materiais utilizados para a moldagem de seis corpos de provas cilíndricos com $50 \mathrm{~mm}$ de diâmetro e $100 \mathrm{~mm}$ de altura pode ser observada no Quadro 4. Ao total, foram moldados doze corpos de 
prova por traço. Para todos os microconcretos resultarem na mesma relação água/aglomerante, foi descontada a quantidade de água da NS coloidal.

Quadro 4- Quantitativo de materiais utilizados para moldagem de seis corpos de prova cilíndricos $50 \times 100 \mathrm{~mm}$ de microconcreto

\begin{tabular}{|c|c|c|c|c|c|c|c|c|c|}
\hline \multirow{3}{*}{$\begin{array}{l}\text { MICROCON- } \\
\text { CRETO }\end{array}$} & \multicolumn{9}{|c|}{ MATERIAL } \\
\hline & \multirow{2}{*}{$\begin{array}{l}\text { CP V- } \\
\text { ARI (g) }\end{array}$} & \multirow{2}{*}{$\begin{array}{l}\text { Nanos } \\
\text { sílica } \\
(g)^{*}\end{array}$} & \multicolumn{4}{|c|}{ Areia (g) } & \multicolumn{2}{|c|}{$\begin{array}{c}\text { Aditivo } \\
\text { superplastificant } \\
\text { e }\end{array}$} & \multirow{2}{*}{$\begin{array}{c}\text { Água } \\
\text { (g) }\end{array}$} \\
\hline & & & Fina & $\begin{array}{c}\text { Média } \\
\text { fina }\end{array}$ & $\begin{array}{l}\text { Média } \\
\text { grossa }\end{array}$ & Grossa & (g) & (\%) & \\
\hline REF & 624,0 & 0,00 & 468,0 & 468,0 & 468,0 & 468,0 & 12,48 & 2 & 300,00 \\
\hline $2 \mathrm{NS}$ & 611,5 & 12,23 & 468,0 & 468,0 & 468,0 & 468,0 & 12,23 & 2 & 291,43 \\
\hline $4 N S$ & 599,0 & 23,96 & 468,0 & 468,0 & 468,0 & 468,0 & 11,98 & 2 & 283,22 \\
\hline $6 \mathrm{NS}$ & 586,6 & 35,19 & 468,0 & 468,0 & 468,0 & 468,0 & 11,73 & 2 & 275,37 \\
\hline $8 N S$ & 574,1 & 45,93 & 468,0 & 468,0 & 468,0 & 468,0 & 11,48 & 2 & 267,84 \\
\hline $10 \mathrm{NS}$ & 561,6 & 56,16 & 468,0 & 468,0 & 468,0 & 468,0 & 11,23 & 2 & 260,69 \\
\hline
\end{tabular}

* A quantidade de NS adicionada à mistura refere-se a NS coloidal (70\% líquido e $30 \%$ NS), resultando no teor efetivo de NS desejado após descontar a quantidade de água na suspensão aquosa.

O procedimento de mistura dos microconcretos seguiu a metodologia descrita na NBR 7215 (ABNT, 2019). Primeiro, foi realizada a adição de água, NS e aditivo superplastificante em uma cuba de aço inox. Depois, foi acrescentado o cimento Portland. Com o auxílio de um cronômetro, foi iniciado a contagem a partir do momento que o cimento entrou em contato com a água. A mistura desses materiais foi feita na velocidade baixa por de $30 \mathrm{~s}$. Logo após esse tempo, sem desligar o misturador planetário, foi adicionada a areia (quatro frações de 468 previamente misturadas) que foram sendo colocadas aos poucos durante os $30 \mathrm{~s}$. Em seguida foi aumentada a velocidade para alta e manteve-se o misturador planetário ligado por mais 30s. Posteriormente, o misturador foi deslitado por $90 \mathrm{~s}$, sendo nos primeiros $30 \mathrm{~s}$ retirado o microconcreto que ficou aderido às paredes da cuba com o auxílio de uma espátula. Nos 60 s restantes a mistura foi deixada em repouso e logo após esse intervalo o misturador foi ligado novamente em velocidade alta por 60s.

Após o preparo dos microconcretos, foi realizada a moldagem dos corpos de prova. O microconcreto foi colocado nas formas com ajuda da espátula, em quatro camadas de alturas aproximadamente iguais, e com cada camada recebendo 30 golpes uniformes e homogeneamente distribuídos com o soquete normal. Logo após a moldagem, os corpos de prova foram cobertos com uma lona plástica por 24 horas para evitar a evaporação de água e posteriormente foram colocados em cura submersa de água saturada de cal até a idade de ensaio.

\subsection{Avaliação das propriedades dos microconcretos}

No concreto endurecido foram realizados ensaios de resistência à compressão conforme a NBR 7215 (ABNT, 2019) aos 1, 3, 7 e 14 dias de hidratação. Foram feitos três corpos de prova por traço para cada idade. Após adquirir os resultados, foi efetuada uma análise de variância ANOVA para cada idade separadamente, com o propósito de avaliar se a substituição parcial do cimento Portland pela NS resultou em diferenças significativas de desempenho mecânico em cada idade. Para isso, foi utilizado o software Statistica v.10, considerando nível de significância 0,05. Logo após realizar esse teste estatístico, foi realizado o teste de Duncan no mesmo software, com intervalo de confiança de 95\%, para agrupar os microconcretos em grupos homogêneos e heterogêneos de resistência à compressão levando em consideração a média e o desvio padrão das amostras ensaiadas. 


\section{Resultados e Discussão}

3.1 Efeito do teor de NS nos microconcretos ao longo do tempo

Os resultados de resistência à compressão dos microconcretos aos 1, 3, 7 e 14 dias são mostrados na Figura 1.

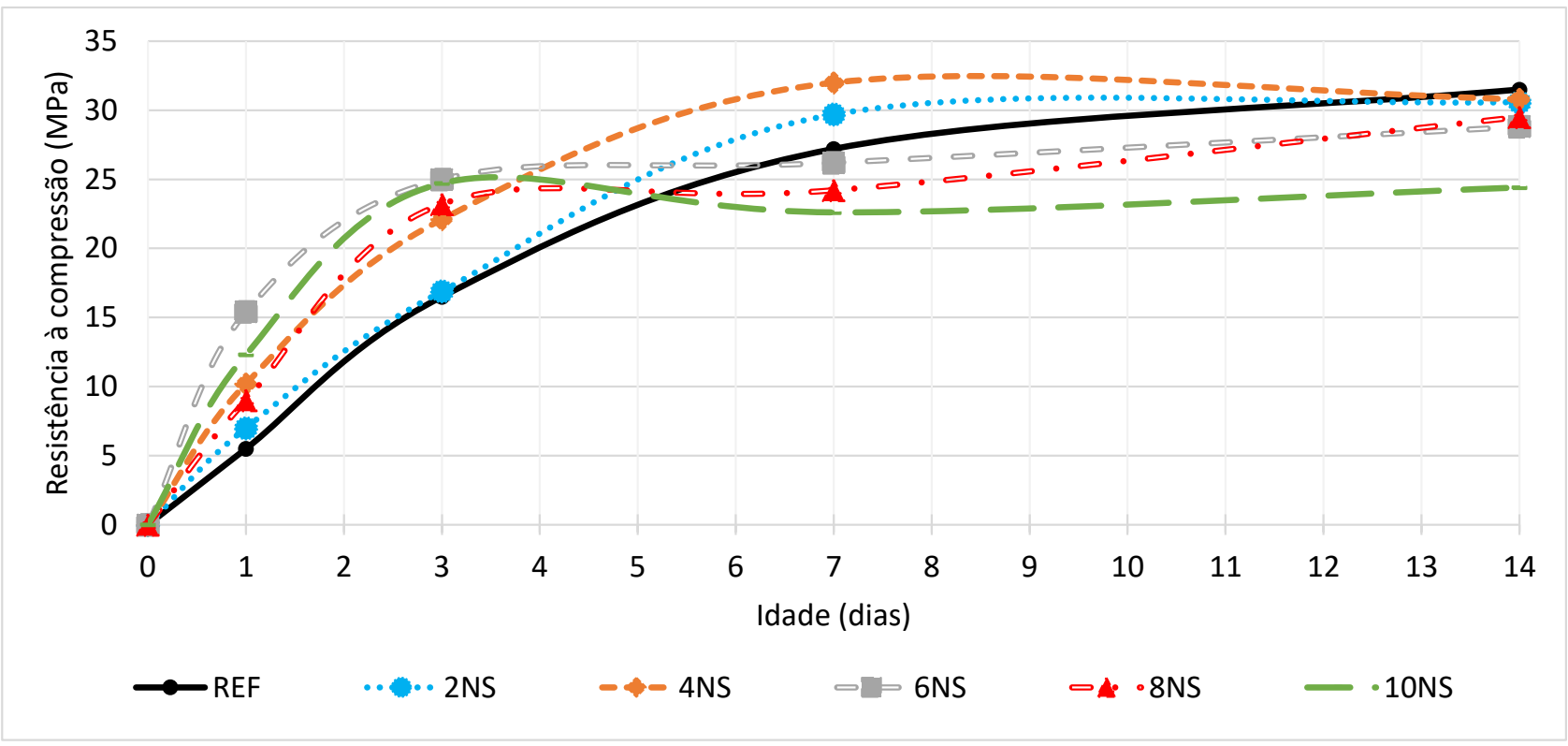

Figura 1 - Resistência à compressão dos microconcretos aos 1, 3, 7 e 14 dias de hidratação

Através dos resultados de resistência à compressão mostrados na Figura 1, observa-se que houve diferença no desempenho mecânico com a utilização de diferentes teores de NS. Pode-se notar que houve semelhança na resistência à compressão dos traços REF e $2 \mathrm{NS}$ em todas as idades avaliadas. Apesar disso, os demais teores de NS resultaram no aumento do desempenho mecânico do microconcreto em relação ao REF até os três dias de hidratação. O comportamento observado está de acordo com a literatura, que justifica o aumento do desempenho mecânico de compósitos cimentícios com a adição de NS em função do aumento do teor de C-S-H e do refinamento da estrutura porosa. Apesar disso, o teor de $2 \%$ de NS resultou em resistência à compressão semelhante ao microconcreto sem adição de NS, divergindo da literatura que mostra que mesmo teores menores podem contribuir para o aumento da resistência à compressão nas idades iniciais (FRAGA et al., 2020; SENFF et al., 2010; SINGH et al., 2012).

Outro comportamento que pode ser destacado na Figura 1 é que para teores maiores de NS (acima de 6\%) houve tendência de estabilização da resistência à compressão a partir dos 3 dias de hidratação. Esse comportamento pode ser justificado devido ao aumento da retração com o aumento do teor de NS, que pode resultar no surgimento de fissuras e, consequentemente, na redução da resistência do compósito cimentício (SILVA JÚNIOR, 2017).

3.2 Análise estatística da resistência à compressão dos microconcretos

Os resultados da análise de variância são mostrados no Quadro 5. 
Quadro 5 - Análise de variância (one-way ANOVA) da resistência à compressão axial dos microconcretos aos 1, 3, 7 e 14 dias de hidratação

\begin{tabular}{|c|c|c|c|c|c|}
\hline Idade & SQ & MQ & $\mathbf{F}$ & p-valor & Resultado \\
\hline 1 dia & 197,489 & 39,497 & 41,969 & $3,42 \mathrm{E}-7$ & Significativo \\
\hline 3 dias & 216,531 & 43,306 & 36,511 & $7,44 \mathrm{E}-7$ & Significativo \\
\hline 7 dias & 182,169 & 36,433 & 11,055 & $3,6 \mathrm{E}-4$ & Significativo \\
\hline 14 dias & 98,677 & 19,735 & 3,391 & $3,85 \mathrm{E}-2$ & Significativo \\
\hline \multicolumn{6}{|c|}{$\begin{array}{l}\text { Sendo: } \\
\text { SQ= Soma dos quadrados; } \\
\text { MQ= Média dos quadrados; } \\
\text { F= Parâmetro de Fisher para o teste de significância; } \\
\text { p-valor= probabilidade de significância. }\end{array}$} \\
\hline
\end{tabular}

Os resultados da análise de variância (one-way ANOVA) mostraram que houve diferença significativa na resistência à compressão do microconcreto em todas as idades analisadas, pois em cada idade o $p$-valor foi menor que o nível de significância de 0,05. Dessa forma, foi realizado o teste de Duncan, conforme mostrado no Quadro 6.

Quadro 6 - Resultados do teste de Duncan para resistência à compressão dos microconcretos aos 1,3, 7 e 14 dias de hidratação

\begin{tabular}{|c|c|c|c|c|c|c|c|}
\hline Idade & Microconcreto & $\begin{array}{l}\text { Resistência } \\
\text { média à } \\
\text { compressão } \\
\text { (MPa) }\end{array}$ & $\begin{array}{c}\text { Desvio } \\
\text { padrão } \\
\text { (MPa) }\end{array}$ & Grupo 1 & Grupo 2 & Grupo 3 & Grupo 4 \\
\hline \multirow{6}{*}{1 dia } & REF & 5,5 & 0,5 & $x$ & & & \\
\hline & $2 \mathrm{NS}$ & 7,0 & 0,1 & $X$ & & & \\
\hline & 4NS & 10,2 & 0,3 & & $X$ & & \\
\hline & $6 \mathrm{NS}$ & 15,4 & 0,4 & & & & $X$ \\
\hline & $8 N S$ & 9,0 & 2,1 & & $X$ & & \\
\hline & 10NS & 12,3 & 0,9 & & & $X$ & \\
\hline \multirow{6}{*}{3 dias } & REF & 16,5 & 1,3 & $x$ & & & \\
\hline & $2 \mathrm{NS}$ & 16,9 & 0,9 & $x$ & & & \\
\hline & 4NS & 22,1 & 0,4 & & $X$ & & \\
\hline & $6 \mathrm{NS}$ & 25,0 & 1,6 & & & $x$ & \\
\hline & $8 N S$ & 23,2 & 0,9 & $x$ & $x$ & & \\
\hline & 10NS & 24,7 & 1,0 & & & $X$ & \\
\hline \multirow{6}{*}{7 dias } & REF & 27,2 & 1,2 & & $x$ & $x$ & \\
\hline & $2 \mathrm{NS}$ & 29,7 & 3,2 & & & $x$ & $x$ \\
\hline & 4NS & 32,0 & 2,6 & & & & $x$ \\
\hline & $6 \mathrm{NS}$ & 26,2 & 0,6 & & & $x$ & \\
\hline & $8 \mathrm{NS}$ & 24,2 & 0,3 & $x$ & $x$ & & \\
\hline & $10 N S$ & 22,6 & 0,6 & $x$ & & & \\
\hline \multirow{6}{*}{14 dias } & REF & 31,5 & 4,6 & & $x$ & & \\
\hline & $2 \mathrm{NS}$ & 30,6 & 2,0 & & $x$ & & \\
\hline & $4 \mathrm{NS}$ & 30,8 & 1,3 & & $x$ & & \\
\hline & $6 \mathrm{NS}$ & 28,8 & 1,1 & & $x$ & & \\
\hline & $8 \mathrm{NS}$ & 29,5 & 0,7 & & $x$ & & \\
\hline & $10 \mathrm{NS}$ & 24,4 & 2,4 & $x$ & & & \\
\hline
\end{tabular}


Com 1 dia de hidratação, foram formados 4 grupos de resistência, sendo o grupo 1 de menor resistência e o grupo 4 de maior resistência. Nessa idade, não houve diferença significativa no desempenho mecânico entre os microconcretos REF e $2 \mathrm{NS}$, que resultaram na menor resistência à compressão entre todos os traços avaliados. Os microconcretos 4NS e 8NS foram englobados no grupo 2, o 10 NS no grupo 3 e o 6 NS no grupo 4, sendo este o de maior desempenho mecânico nesta idade.

Aos 3 dias de hidratação foram formados 3 grupos de resistência, sendo o grupo 1 de menor resistência e o grupo 3 de maior resistência. Assim como com 1 dia de hidratação, aos 3 dias os microconcretos REF e 2NS resultaram no mesmo desempenho mecânico e os demais teores de NS contribuíram para o aumento do desempenho mecânico dos microconcretos 4NS, 6NS, 8NS e 10NS em relação ao microconcreto REF.

Aos 7 dias de hidratação, foram formados 4 grupos de resistência. Nessa idade, observa-se que os teores de $8 \%$ e $10 \%$ de NS foram prejudiciais ao desempenho mecânico do concreto devido à retração que superou o efeito benéfico da reação pozolânica. Nessa idade, destacou-se o teor de $4 \%$ de NS que resultou no maior desempenho mecânico.

Aos 14 dias de hidratação foram formados apenas 2 grupos, sendo o grupo 1 de menor resistência e o grupo 2 de maior resistência. Todos os microconcretos foram encaixados no grupo 2 , exceto o microconcreto $10 \mathrm{NS}$ que foi classificado no grupo 1 . Dessa forma, nessa idade não houve diferença no desempenho mecânico do microconcreto REF e dos microconcretos com $2 \%, 4 \%, 6 \%$ e $8 \%$ de NS, porém quando foi utilizado o teor de $10 \%$ de NS houve diminuição do desempenho mecânico em relação aos demais traços.

Ressalta-se que mesmo com menor quantidade de cimento os microconcretos contendo NS, em geral, resultaram no aumento do desempenho mecânico dos microconcretos. Esse comportamento é justificado pela reação pozolânica da NS com o CH oriundo das reações de hidratação do cimento Portland, formando C-S-H adicional, que preenche os vazios capilares, densificando a microestrutura do compósito cimentício e refinando a estrutura porosa (LI et al., 2017; NOLI, 2015). A redução do desempenho mecânico com maiores teores de NS é justificado pelo efeito negativo que a NS possui no aumento da retração (SILVA JÚNIOR, 2017).

\section{Conclusões}

Esta pesquisa teve como objetivo analisar a influência da substituição parcial do cimento Portland por diferentes teores de nanossílica no desempenho mecânico do microconcreto. Foi constatado que não houve efeito significativo ao utilizar $2 \%$ de NS na resistência à compressão do microconcreto nas idades estudadas.

Além disso, foi observado que conforme houve um aumento no teor de NS, o microconcreto teve aumento do desempenho mecânico nas idades iniciais e sua redução em idades maiores, fatos que podem ser atribuídos à reação pozolânica e à retração, respectivamente.

Dentre os teores estudados, o teor de $4 \%$ de NS mostrou-se bastante promissor. A curva de resistência em função do tempo do traço 4NS, apresentou crescimento gradual e equilibrado, com resistências superiores aos demais traços aos 7 dias de hidratação e estabilização aos 14 dias. Ao decorrer do tempo, as resistências de todos os traços foram coincidindo com a curva da referência, ou seja, o efeito pozolânico ao decorrer do tempo foi sendo compensado com as altas retrações neles obtidos. A partir dos resultados obtidos nesta pesquisa, sugere-se para estudos futuros:

- analisar a influência de diferentes tipos de NS nas propriedades de compósitos cimentícios;

- verificar o efeito de diferentes teores de NS no consumo de CH e formação de C-S-H adicional em pastas de cimento;

- estudar o efeito de diferentes teores de NS na retração de microconcretos;

- avaliar a durabilidade do concreto produzido com diferentes teores de NS.

\section{Referências Bibliográficas}

Abd.El.Aleem, S.; Heikal, M.; Morsi, W. M. (2014). Hydration characteristic, thermal expansion and microstructure of cement containing nano-silica. Construction and Building Materials, v. 59, p. 151-160.

ABNT, Associação Brasileira de Normas Técnicas. (2019). NBR 7215: Cimento Portland - Determinação da resistência à compressão de corpos de prova cilíndricos. Rio de Janeiro. 
Flores, Y. C.; Cordeiro, G. C.; Toledo Filho, R. D.; Tavares, L. M. (2017). Performance of Portland cement pastes containing nano-silica and different types of silica. Construction and Building Materials, v. 146, p. 524530.

Fraga, Y. S. B.; Rêgo, J. H. S.; Capuzzo, V. M. S.; Andrade, D. S.; Morais, P. C. (2020b). Ultrasonication and synergistic effects of silica fume and colloidal nanosilica on the C-S-H microstructure. Journal of Building Engineering, v. 32, p. 1-13.

Li, L. G.; Huang, Z. H.; Zhu, J.; Kwan, A. K. H.; Chen, H. Y. (2017). Synergistic effects of micro-silica and nanosilica on strength and microstructure of mortar. Construction and Building Materials, v. 140, p. 229-238.

Noli, P. R. F. O. (2015). Propriedades mecânicas do concreto de alto desempenho com adição de nanosílica estabilizada. Tese (Mestrado em Estruturas e Construção Civil) - Universidade Federal de Minas Gerais, Minas Gerais.

Senff, L.; Hotza, D.; Repette, W. L.; Ferreira, V. M.; Labrincha, J. A. (2010). Effect of nanosilica and microsilica on microstructure and hardened properties of cement pastes and mortars. Advances in Applied Ceramics, v. 109 , n. 2, p. 104-110.

Silva Júnior, P. F. (2017). Estudo da estabilidade dimensional de concretos de alta resistência com adição de polímero superabsorvente e nanopartículas de sílica. Tese (Doutorado em Estruturas e Construção Civil) Universidade de Brasília, Distrito Federal.

Singh, L. P.; Bhattacharyya, S. K.; Ahalawat, S. (2012). Preparation of size controlled silica nano particles and its functional role in cementitious system. Journal of Advanced Concrete Technology, v. 10, n. 11, p. 345352.

Vaske, N. R.; Campagnolo, J. L.; Dal Molin, D. C. C. (2010). Relationship between the compressive strength of silica fume mortar applied to the substratum and the one obtained in standardized cylindrical test specimens. Revista Ibracon de Estruturas e Materiais, v. 3, n. 3, p. 322-345. 